\title{
OPERATOR ALGEBRAS RELATED TO THOMPSON'S GROUP $\boldsymbol{F}$
}

\author{
PAUL JOLISSAINT
}

(Received 24 October 2003; revised 25 November 2004)

Communicated by A. Pryde

\begin{abstract}
Let $F^{\prime}$ be the commutator subgroup of $F$ and let $\Gamma_{0}$ be the cyclic group generated by the first generator of $F$. We continue the study of the central sequences of the factor $L\left(F^{\prime}\right)$, and we prove that the abelian von Neumann algebra $L\left(\Gamma_{0}\right)$ is a strongly singular MASA in $L(F)$. We also prove that the natural action of $F$ on $[0,1]$ is ergodic and that its ratio set is $\{0] \cup\left\{2^{k} ; k \in \mathbb{Z}\right\}$.
\end{abstract}

2000 Mathematics subject classification: primary 37A10; secondary 43A07.

\section{Introduction}

Thompson's group $F$ is a countable group with infinite conjugacy classes which has remarkable properties, discovered in 1965 and rediscovered later by homotopy theorists. It is known that $F$ contains no non abelian free subgroup, and it is still unknown whether it is amenable or not. However, it shares many properties with amenable groups; for instance, it is inner amenable: see [6, 7].

We recall the two most commonly known descriptions of $F$. On the one hand, as an abstract group, it has the following presentation:

$$
F=\left\langle x_{0}, x_{1}, \ldots \mid x_{i}^{-1} x_{n} x_{i}=x_{n+1}, 0 \leq i<n\right\rangle .
$$

On the other hand, it is the group of homeomorphisms of the interval $[0,1]$ that are piecewise linear, differentiable except at finitely many dyadic rational numbers and such that on intervals of differentiability the derivatives are integral powers of 2 . To each of these descriptions corresponds a tool that we present below. We refer to $[1,2]$ for more details on Thompson's groups $F, T$ and $V$, and for some results that will be

(C) 2005 Australian Mathematical Society 1446-7887/05 $\$ A 2.00+0.00$ 
used here. The first one is the existence of a unique normal form for every non trivial element of $F$; it is Corollary-Definition 2.7 of [2].

LEMMA 1.1. Every non trivial element of $F$ can be expressed in unique normal form $x_{i_{1}} \cdots x_{i_{k}} x_{j_{m}}^{-1} \cdots x_{j_{1}}^{-1}$, where

(1) $0 \leq i_{1} \leq \cdots \leq i_{k}, j_{1} \leq \cdots \leq j_{m}$ and $i_{k} \neq j_{m}$;

(2) if $x_{i}$ and $x_{i}^{-1}$ appear explicitly in the expression for some $i$, then also does $x_{i+1}$ or $x_{i+1}^{-1}$.

The second lemma that will be needed is related to the action of $F$ on $[0,1]$ : see [2, Lemma 4.2].

LEMMA 1.2. If $0=a_{0}<a_{1}<\cdots<a_{n}=1$ and $0=b_{0}<b_{1}<\cdots<b_{n}=1$ are partitions of $[0,1]$ consisting of dyadic rational numbers, then there exists $g \in F$ such that $g\left(a_{i}\right)=b_{i}$ for every $i=0, \ldots, n$. If moreover $a_{i-1}=b_{i-1}$ and $a_{i}=b_{i}$ for some $i>0$, then $g$ can be chosen so that $g(t)=t$ for every $t \in\left[a_{i-1}, a_{i}\right]$.

In [7], we studied the von Neumann algebras associated with $F$ and with some of its subgroups: we proved not only that the factor $L(F)$ is a McDuff factor (that is, that $L(F)$ is *-isomorphic to the tensor product factor $L(F) \otimes R$ where $R$ denotes the hyperfinite $\mathrm{II}_{1}$ factor), but that the pair $L\left(F^{\prime}\right) \subset L(F)$ has the relative McDuff property: there is a *isomorphism $\Phi: L(F) \otimes R \rightarrow L(F)$ such that its restriction to $L\left(F^{\prime}\right) \otimes R$ is still an isomorphism onto the subfactor $L\left(F^{\prime}\right)$ associated to the derived subgroup $F^{\prime}=[F, F]$ of $F$.

In Section 2, we present further properties of central sequences of $L\left(F^{\prime}\right):$ on the one hand, we prove that there exists a sequence of unitary operators $\left(u_{n}\right) \subset L\left(F^{\prime}\right)$ such that $\left(u_{n} x u_{n}^{*}\right)$ is a central sequence for every $x \in L\left(F^{\prime}\right)$, and on the other hand, we show that $L\left(F^{\prime}\right)$ is approximately normal in $L(F)$ in the sense of [3].

Section 3 is devoted to the study of the von Neumann subalgebra $L\left(\Gamma_{0}\right)$ where $\Gamma_{0}$ is the cyclic subgroup of $F$ generated by the generator $x_{0}: L\left(\Gamma_{0}\right)$ is a strongly singular MASA of $L(F)$ (see [8]) and we show that its Pukanszky invariant is $\{\infty\}$.

Finally, we prove in Section 4 that the natural action of $F$ on $[0,1]$ is ergodic and we show that its ratio set is $\{0\} \cup\left\{2^{k} ; k \in \mathbb{Z}\right\}$.

Notation For $g \in F$ viewed as a homeomorphism of $[0,1]$, we denote by $S(g)$ the set of numbers $t \in[0,1]$ such that $g(t) \neq t$. We will use the following properties which are very easy to prove:

(S1) $S\left(g^{-1}\right)=S(g)$ for every $g \in F$;

(S2) if $t \in S(g)$ then $g(t) \in S(g)$ for every $g \in F$;

(S3) $S\left(g h g^{-1}\right)=g(S(h))$ for all $g, h \in F$;

(S4) if $S(g) \cap S(h)=\emptyset$, then $g h=h g$. 
Let $M$ be a finite von Neumann algebra and let $\tau$ be a normal, normalized, faithful trace on $M$. We denote by $\|a\|_{2}=\tau\left(a^{*} a\right)^{1 / 2}$ the associated Hilbertian norm.

If $\Gamma$ is a countable group, let $\lambda$ denote its left regular representation on $\ell^{2}(\Gamma)$ defined by:

$$
(\lambda(g) \xi)(h)=\xi\left(g^{-1} h\right)
$$

for all $g, h \in \Gamma$ and $\xi \in \ell^{2}(\Gamma)$. The bicommutant $\lambda(\Gamma)^{\prime \prime}$ in the algebra of linear, bounded operators on $\ell^{2}(\Gamma)$ is a von Neumann algebra denoted by $L(\Gamma)$. It is a finite von Neumann algebra with natural trace $\tau$ defined by $\tau(x)=\left\langle x \delta_{e}, \delta_{e}\right\rangle$, where $\delta_{e}$ is the characteristic function of $\{e\} . L(\Gamma)$ is a type $I_{1}$ factor if and only if $\Gamma$ is an icc group. Every operator $x \in L(\Gamma)$ is expressed as a series $\sum_{g \in \Gamma} x(g) \lambda(g)$ where $x(g)=\tau\left(x \lambda\left(g^{-1}\right)\right)$ and $\sum_{g \in \Gamma}|x(g)|^{2}=\|x\|_{2}^{2}$. If $H$ is a subgroup of $\Gamma$, then $L(H)$ is identified in a natural way as a von Neumann subalgebra of $L(\Gamma)$ : it is the subset of elements $x$ such that $x(g)=0$ for every $g \notin H$.

Let $M$ be a type $\Pi_{1}$ factor. A central sequence in $M$ is a bounded sequence $\left(x_{n}\right) \subset M$ such that $\left\|x_{n} y-y x_{n}\right\|_{2} \rightarrow 0$ as $n \rightarrow \infty$ for every $y \in M$. We write sometimes $[a, b]$ for $a b-b a$.

\section{The subfactor $L\left(F^{\prime}\right)$ of $L(F)$}

Let $F^{\prime}$ denote the commutator subgroup of $F$ as in the preceding section. It is known that it is a simple group, and it is the subgroup of $F$ of elements that coincide with the identity in neighbourhoods of 0 and 1 . Our first result states that $L\left(F^{\prime}\right)$ is inner asymptotically commutative in the sense of [10].

PROPOSITION 2.1. There exists a sequence $\left(u_{n}\right)_{n \geq 1} \subset U\left(L\left(F^{\prime}\right)\right)$ such that

$$
\lim _{n \rightarrow \infty}\left\|\left[u_{n} x u_{n}^{*}, y\right]\right\|_{2}=0
$$

for all $x, y \in L\left(F^{\prime}\right)$. In other words, for every $x \in L\left(F^{\prime}\right),\left(u_{n} x u_{n}^{*}\right)$ is a central sequence of $L\left(F^{\prime}\right)$.

We don't know if $L(F)$ itself is inner asymptotically commutative.

The proof rests on the following lemma of Zeller-Meyer:

LEMMA 2.2. Let $G$ be an icc group. If there exists an increasing sequence $\left(E_{n}\right)$ of subsets of $G$ and a sequence $\left(g_{n}\right)$ of elements of $G$ such that $\bigcup E_{n}=G$ and $g_{n} g g_{n}^{-1} h=h g_{n} g g_{n}^{-1}$ for all $g, h \in E_{n}$ and for every $n$, then the factor $L(G)$ is inner asymptotically commutative. 
PROOF OF PROPOSITION 2.1. Set, for $n \geq 1$,

$$
E_{n}=\left\{g \in F^{\prime} ; S(g) \subset\left(1 / 2^{n}, 1-1 / 2^{n}\right)\right\},
$$

so that $E_{n} \subset E_{n+1}$ and $\bigcup_{n} E_{n}=F^{\prime}$.

Fix $n \geq 1$ and consider the following dyadic partitions of $[0,1]$ :

and

$$
0=a_{0}<a_{1}=\frac{1}{2^{n+1}}<a_{2}=\frac{1}{2^{n}}<a_{3}=1-\frac{1}{2^{n}}<a_{4}=1-\frac{1}{2^{n+1}}<a_{5}=1
$$

$$
0=b_{0}<b_{1}=\frac{1}{2^{n+1}}<b_{2}=1-\frac{1}{2^{n}}<b_{3}=1-\frac{3}{2^{n+2}}<b_{4}=1-\frac{1}{2^{n+1}}<b_{5}=1
$$

so that $b_{3}$ is the mean value of $1-1 / 2^{n}$ and $1-1 / 2^{n+1}$. By Lemma 1.2 , one can find $g_{n} \in F$ such that $g_{n}\left(a_{i}\right)=b_{i}$ for $i=0, \ldots, 5$ and that $g_{n}(t)=t$ for $t \in\left[0, a_{1}\right] \cup\left[a_{4}, 1\right]$. Thus $g_{n} \in F^{\prime}$.

Finally, if $x, y \in E_{n}$, Property (S3) of Section 1 gives

$$
S\left(g_{n} \times g_{n}^{-1}\right)=g_{n}(S(x)) \subset g_{n}\left(\left(a_{2}, a_{3}\right)\right) \subset\left(b_{2}, b_{3}\right),
$$

and as $S(y) \subset\left(a_{2}, a_{3}\right)$, Property (S4) yields $g_{n} x g_{n}^{-1} y=y g_{n} x g_{n}^{-1}$. Lemma 2.2 implies that $L\left(F^{\prime}\right)$ is inner asymptotically commutative.

Before stating the next proposition, let $M$ be a type $\mathrm{II}_{1}$ factor and let $N$ be a subalgebra of $M . N$ is called normal in $M$ if $\left(N^{\prime} \cap M\right)^{\prime} \cap M=N$. Following [3], we say that $N$ is approximately normal in $M$ if

$$
\begin{gathered}
N=\left\{x \in M ;\left\|y_{n} x-x y_{n}\right\|_{2} \rightarrow 0\right. \text { for all bounded sequences } \\
\left.\left(y_{n}\right) \subset M \text { such that }\left\|y_{n} y-y y_{n}\right\|_{2} \rightarrow 0 \text { for all } y \in N\right\} .
\end{gathered}
$$

PROPOSITION 2.3. The subfactor $L\left(F^{\prime}\right)$ is approximately normal in $L(F)$.

For future use, denote by $\mathscr{T}$ the set of sequences $\left(g_{n}\right)_{n \geq 1} \subset F^{\prime}$ such that, for every $\varepsilon>0$, there exists an integer $l>0$ such that $S\left(g_{n}\right) \cap[\varepsilon, 1-\varepsilon]=\emptyset$ for every $n>l$. In fact, if $\left(g_{n}\right) \in \mathscr{T}$, then the sequence $\left(\lambda\left(g_{n}\right)\right) \subset L\left(F^{\prime}\right)$ is a central sequence (see for instance the proof of [7, Proposition 2.4]).

The proof of Proposition 2.3 rests on the following lemma:

LEMmA 2.4. Let $h \in F \backslash F^{\prime}$. Then there exists $\left(g_{n}\right) \in \mathscr{T}$ such that the set $\left\{g_{n} h g_{n}^{-1} ; n \geq 1\right\}$ is infinite.

Proof. Since $h \notin F$, its right derivative at 0 or its left derivative at 1 is equal to $2^{k}$ for some non zero integer $k$. Assume to begin with that there exist $\varepsilon>0$ and a positive 
integer $k$ such that $h(t)=2^{k} t$ for $t \in[0, \varepsilon]$. Choose an integer $l \geq 1$ big enough so that $3 / 2^{l+2} \leq \varepsilon$. For $n \geq l$, set $a_{n}=1 / 2^{k+n+1}, b_{n}=3 / 2^{k+n+2}, c_{n}=1 / 2^{k+n}$ and

$$
a_{n}<b_{n}^{\prime}=\frac{3}{2^{k+n+2}}-\frac{1}{2^{k+n+3}}=\frac{5}{2^{k+n+3}}<b_{n} .
$$

There exists $g_{n} \in F^{\prime}$ such that $g_{n}(t)=t$ for $t \in\left[0, a_{n}\right] \cup\left[c_{n}, 1\right]$ and $g_{n}\left(b_{n}\right)=b_{n}^{\prime}$. Thus, setting $g_{n}=e$ for $n \leq l$, we get that $\left(g_{n}\right) \in \mathscr{T}$. Finally, let us check that if $m>k+n$, then $g_{m} h g_{m}^{-1} \neq g_{n} h g_{n}^{-1}$. As $S\left(g_{p}\right) \subset\left(1 / 2^{k+p+1}, 1 / 2^{k+p}\right)$, we get, on the one hand,

$$
g_{n} h g_{n}^{-1}\left(b_{m}^{\prime}\right)=g_{n} h\left(b_{m}^{\prime}\right)=g_{n}\left(2^{k} b_{m}^{\prime}\right)=\frac{5}{2^{m+3}}
$$

since $5 / 2^{m+3}<1 / 2^{k+n+1}$. On the other hand, the same kind of argument using $3 / 2^{m+3} \leq \varepsilon$ shows that

$$
g_{m} h g_{m}^{-1}\left(b_{m}^{\prime}\right)=\frac{3}{2^{m+3}} \neq g_{n} h g_{n}^{-1}\left(b_{m}^{\prime}\right)
$$

If $h(t)=2^{-k} t$ near 0 with $k>0$, then apply the above arguments to $h^{-1}$. Finally, if $h(t)=t$ near 0 , we reduce to the above cases in using the order two automorphism $\theta$ of $F$ defined by $\theta(g)(t)=1-g(1-t)$ : apply the above arguments to $\theta(h)$ and take the sequence $\left(\theta\left(g_{n}\right)\right) \in \mathscr{T}$. This ends the proof of the lemma.

ProOf OF Proposition 2.3. We only need to show that if $x \in L(F)$ is such that $\left\|\left[x, \lambda\left(g_{n}\right)\right]\right\|_{2} \rightarrow 0$ as $n \rightarrow \infty$ for every sequence $\left(g_{n}\right) \in \mathscr{T}$, then $x \in L\left(F^{\prime}\right)$.

Let $h \in F \backslash F^{\prime}$; we will prove that $x(h)=0$. By the previous lemma, one can find a sequence $\left(g_{n}\right) \in \mathscr{T}$ such that $g_{n} h g_{n}^{-1} \neq g_{m} h g_{m}^{-1}$ for all $n \neq m$.

Fix $\varepsilon>0$. There exists an integer $l \geq 1$ such that $\left\|x-\lambda\left(g_{n}^{-1}\right) x \lambda\left(g_{n}\right)\right\|_{2}<\varepsilon / 2$ for $n \geq l$. Moreover, the series

$$
\sum_{n=1}^{\infty}\left|x\left(g_{n} h g_{n}^{-1}\right)\right|^{2}
$$

converges. Hence one can find an integer $l^{\prime} \geq l$ such that $\left|x\left(g_{n} h g_{n}^{-1}\right)\right|<\varepsilon / 2$ for every $n \geq l^{\prime}$. Then we have, for any $n \geq l^{\prime}$,

$$
\begin{aligned}
|x(h)| & =\left|\tau\left(x \lambda\left(h^{-1}\right)\right)\right| \\
& \leq\left|\tau\left(x \lambda\left(h^{-1}\right)-x \lambda\left(g_{n} h^{-1} g_{n}^{-1}\right)\right)\right|+\left|\tau\left(x \lambda\left(g_{n} h^{-1} g_{n}^{-1}\right)\right)\right| \\
& =\left|\tau\left(x \lambda\left(h^{-1}\right)\right)-\tau\left(\lambda\left(g_{n}^{-1}\right) x \lambda\left(g_{n}\right) \lambda\left(h^{-1}\right)\right)\right|+\left|\tau\left(x \lambda\left(g_{n} h^{-1} g_{n}^{-1}\right)\right)\right| \\
& \leq\left\|x-\lambda\left(g_{n}^{-1}\right) x \lambda\left(g_{n}\right)\right\|_{2}+\left|x\left(g_{n} h g_{n}^{-1}\right)\right|<\varepsilon .
\end{aligned}
$$

Thus $x(h)=0$. 


\section{A strongly singular MASA in $L(F)$}

Let $\Gamma$ be an icc countable group and let $\Gamma_{0}$ be an abelian subgroup of $\Gamma$. When $\Gamma=F, \Gamma_{0}$ denotes exclusively the cyclic subgroup generated by $x_{0} \in F$.

Recall from [8] that an abelian von Neumann subalgebra $A$ of a type $\mathrm{II}_{1}$ factor $M$ is strongly singular if the following inequality is true for every unitary element $u \in M$ :

$$
\left\|E_{u A u^{*}}-E_{A}\right\|_{\infty, 2} \geq\left\|u-E_{A}(u)\right\|_{2},
$$

where $E_{B}$ denotes the trace-preserving conditional expectation from $M$ onto the von Neumann algebra $B$, and, for $\phi: M \rightarrow M$ linear,

$$
\|\phi\|_{\infty, 2}=\sup \left\{\|\phi(x)\|_{2} ;\|x\| \leq 1\right\} .
$$

Observe that such an algebra is automatically a MASA of $M$. When $M=L(\Gamma)$ is a factor associated to an icc group $\Gamma$ and $A=L\left(\Gamma_{0}\right)$ where $\Gamma_{0}$ is an abelian subgroup of $\Gamma,[8$, Lemma 4.1$]$ gives a sufficient condition in order that $A$ be a strongly singular MASA in $M$; we recall its statement for convenience.

LEMMA 3.1 ([8]). Let $\Gamma$ be an icc group with an abelian subgroup $\Gamma_{0}$. Assume that they satisfy the following condition:

(SS) If $g_{1}, \ldots, g_{m}, h_{1}, \ldots, h_{n} \in \Gamma \backslash \Gamma_{0}$, then there exists $g_{0} \in \Gamma_{0}$ such that $g_{i} g_{0} h_{j} \notin \Gamma_{0}$ for all $i=1, \ldots, m$ and all $j=1, \ldots, n$.

Then $A=L\left(\Gamma_{0}\right)$ is a strongly singular MASA in $L(\Gamma)$.

It turns out that the abelian subgroup $\Gamma_{0}$ of $F$ satisfies a stronger condition that will be also used to prove an ergodic property for the conditional expectation onto $L\left(\Gamma_{0}\right)$.

LEMMA 3.2. The pair $\left(\Gamma_{0}, F\right)$ satisfies the following condition:

(ST) If $g_{1}, \ldots, g_{m}, h_{1}, \ldots, h_{n} \in F \backslash \Gamma_{0}$, then there exists a finite subset $E$ of $\Gamma_{0}$ such that $g_{i} g_{0} h_{j} \notin \Gamma_{0}$ for all $i=1, \ldots, m$, all $j=1, \ldots, n$ and for every $g_{0} \in \Gamma_{0} \backslash E$.

ProOF. Fix $g_{1}, \ldots, g_{m}, h_{1}, \ldots, h_{n} \in F \backslash \Gamma_{0}$. We write $g_{i}=x_{0}^{p_{i}} g_{i}^{\prime} x_{0}^{-q_{i}}$ and $h_{j}=$ $x_{0}^{r_{j}} h_{j}^{\prime} x_{0}^{-s_{j}}$ for all $i, j$, where $p_{i}, q_{i}, r_{j}$ and $s_{j}$ are non negative integers, and where $g_{i}^{\prime}, h_{j}^{\prime}$ all belong to $F_{1} \backslash\{e\}$, where $F_{1}$ is the subgroup of $F$ generated by $\left\{x_{1}, x_{2}, \ldots\right\}$. It is the range of the 'shift map' $\phi: F \rightarrow F$ defined by $\phi\left(x_{n}\right)=x_{n+1}$ for every $n \geq 0$. See [1, page 369]. Hence $x_{0}^{-k} g x_{0}^{k}=\phi^{k}(g)$ for every positive integer $k$ and every $g \in F_{1}$. Using normal forms of $g_{i}^{\prime}$ and $h_{j}^{\prime}$, observe that if $l$ is a sufficiently large integer, then $\phi^{\prime}\left(g_{i}^{\prime}\right) h_{j}^{\prime} \notin \Gamma_{0}$ for all $i, j$. Thus, if $k>0$ is large enough and if we put $l_{i, j}=k-q_{i}+r_{j}$, we get $g_{i} x_{0}^{k} h_{j}=x_{0}^{p_{i}} g_{i}^{\prime} x_{0}^{l_{i j}} h_{j}^{\prime} x_{0}^{-s_{j}}=x_{0}^{p_{i}+l_{i, j}} \phi^{l_{i j}}\left(g_{i}^{\prime}\right) h_{j}^{\prime} x_{0}^{-s_{j}}$, and we deduce that $g_{i} x_{0}^{k} h_{j}$ does not belong to $\Gamma_{0}$. 
When $k=-k^{\prime}$ is negative, we have $g_{i}^{\prime} x_{0}^{-k^{\prime}} h_{j}^{\prime}=g_{i}^{\prime} \phi^{k^{\prime}}\left(h_{j}^{\prime}\right) x_{0}^{-k^{\prime}}$ for all $i, j$, which gives the same conclusion.

Similarly, it is easy to see that if $\Gamma$ is the free group $F_{N}$ of rank $N \geq 2$ on free generators $a_{1}, \ldots, a_{N}$ and if $\Gamma_{0}$ is the subgroup generated by some $a_{i}$, then the pair $\left(\Gamma_{0}, \Gamma\right)$ satisfies condition (ST).

PROPOSITION 3.3. Assume that $\Gamma_{0}$ is an abelian subgroup of an icc group $\Gamma$ and that the pair $\left(\Gamma_{0}, \Gamma\right)$ satisfies condition (ST). Set $A=L\left(\Gamma_{0}\right)$ and $M=L(\Gamma)$. Then $A$ is a strongly singular MASA in $M$ and the conditional expectation $E_{A}$ satisfies:

$$
E_{A}(x)=w-\lim _{g_{0} \rightarrow \infty} \lambda\left(g_{0}\right) x \lambda\left(g_{0}^{-1}\right)
$$

for every $x \in M$.

PROOF. The first assertion follows from Lemma 3.1. In order to prove the second one, fix $x$ and $y \in M$ such that $\{g \in \Gamma ; x(g) \neq 0\}$ and $\{g \in \Gamma ; y(g) \neq 0\}$ are finite. We will prove that there exists a finite subset $E$ of $\Gamma_{0}$ such that

$$
\tau\left(E_{A}(x) y^{*}\right)=\tau\left(\lambda\left(g_{0}\right) x \lambda\left(g_{0}^{-1}\right) y^{*}\right)
$$

for every $g_{0} \in \Gamma_{0} \backslash E$. We write $C=\left\{g \in \Gamma \backslash \Gamma_{0} ; x(g) \neq 0\right\}$ and $D=\left\{g \in \Gamma \backslash \Gamma_{0}\right.$; $y(g) \neq 0\}$, which are finite sets, and we decompose $x$ and $y$ as $x=E_{A}(x)+x^{\prime}$ and $y=E_{A}(y)+y^{\prime}$ so that $x^{\prime}=\sum_{g \in C} x(g) \lambda(g)$ and $y^{\prime}=\sum_{g \in D} y(g) \lambda(g)$ are orthogonal to $A$.

It is easy to see that condition (ST) implies the existence of a finite subset $E$ of $\Gamma_{0}$ such that $g_{0} C g_{0}^{-1} \cap D=\emptyset$ for every $g_{0} \in \Gamma_{0} \backslash E$. Take $g_{0} \in \Gamma_{0} \backslash E$; since $\Gamma_{0}$ is abelian, we have $\lambda\left(g_{0}\right) E_{A}(x) \lambda\left(g_{0}^{-1}\right)=E_{A}(x)$ and similarly for $y$. Thus

$$
\begin{aligned}
\tau\left(\lambda\left(g_{0}\right) x \lambda\left(g_{0}^{-1}\right) y^{*}\right) & =\tau\left(E_{A}(x) y^{*}\right)+\tau\left(\lambda\left(g_{0}\right) x^{\prime} \lambda\left(g_{0}^{-1}\right) y^{*}\right) \\
& =\tau\left(E_{A}(x) y^{*}\right)+\tau\left(x^{\prime} E_{A}\left(y^{*}\right)\right)+\tau\left(x^{\prime} \lambda\left(g_{0}^{-1}\right) y^{\prime *} \lambda\left(g_{0}\right)\right) \\
& =\tau\left(E_{A}(x) y^{*}\right)
\end{aligned}
$$

since $\tau\left(x^{\prime} E_{A}\left(y^{*}\right)\right)=0$ by orthogonality, and

$$
\begin{aligned}
\tau\left(x^{\prime} \lambda\left(g_{0}^{-1}\right) y^{\prime *} \lambda\left(g_{0}\right)\right) & =\tau\left(\sum_{h, k \in \Gamma \backslash \Gamma_{0}} x(h) \overline{y(k)} \lambda\left(h g_{0}^{-1} k^{-1} g_{0}\right)\right) \\
& =\sum_{h \in C} x(h) \overline{y\left(g_{0} h g_{0}^{-1}\right)}=0
\end{aligned}
$$

because $g_{0} C g_{0}^{-1} \cap D=\emptyset$. 
Our last result about $L\left(\Gamma_{0}\right) \subset L(F)$ is the computation of its Pukanszky invariant which was suggested to us by G. Robertson. Let $A$ be a MASA of some type $\Pi_{1}$ factor $M$. Denote by $\mathscr{A}$ the type I von Neumann algebra generated by $A \cup A^{\prime}$ in the algebra $B\left(L^{2}(M)\right.$ ), where $A^{\prime}$ is the commutant of $A$. The orthogonal projection $e_{A}$ of $L^{2}(M)$ onto $L^{2}(A)$ lies in the centre of $\mathscr{A}^{\prime}$ and the reduced algebra $\mathscr{A}^{\prime} e_{A}$ is abelian. The reduced von Neumann algebra $\mathscr{A}^{\prime}\left(1-e_{A}\right)$ decomposes as a direct sum $\mathscr{A}^{\prime}\left(1-e_{A}\right)=$ $\mathscr{A}_{n_{1}}^{\prime} \oplus \mathscr{A}_{n_{2}}^{\prime} \oplus \ldots$ of homogeneous type $\mathrm{I}_{n_{i}}$ algebras, where $1 \leq n_{1}<n_{2}<\cdots \leq \infty$. Then the Pukanszky invariant of the pair $A \subset M$ is the set $\left\{n_{1}, n_{2}, \ldots\right\}$.

Let us recall $[9$, Proposition 3.6] which gives a way to compute Pukanszky invariant for pairs $L\left(\Gamma_{0}\right) \subset L(\Gamma)$ as above.

PROPOSITION 3.4 ([9]). Suppose that $\Gamma_{0}$ is an abelian subgroup of an icc group $\Gamma$ such that $L\left(\Gamma_{0}\right)$ is a MASA in $L(\Gamma)$. If $g^{-1} \Gamma_{0} g \cap \Gamma_{0}=\{e\}$ for every $g \in \Gamma \backslash \Gamma_{0}$, then the Pukanszky invariant of the pair $L\left(\Gamma_{0}\right) \subset L(\Gamma)$ is the set reduced to the number $n$ of double classes $\Gamma_{0} g \Gamma_{0}$.

PROPOSITION 3.5. The Pukanszky invariant of the pair $L\left(\Gamma_{0}\right) \subset L(F)$ is $\{\infty\}$.

PROOF. There exists a homomorphism $\psi: F \rightarrow \mathbb{Z}$ such that $\psi\left(x_{n}\right)=1$ for every $n \geq 0$ because the defining relations of $F$ are homogeneous. If there would exist $g \in F \backslash \Gamma_{0}$ and $k, l \in \mathbb{Z}$ such that $g x_{0}^{k} g^{-1}=x_{0}^{l}$, then, applying $\psi$ on both sides gives $k=l$ which can be assumed positive. Let us write $g=x_{0}^{p} g^{\prime} x_{0}^{-q}$ with $g^{\prime} \in F_{1} \backslash\{e\}$ as in the proof of Lemma 2.4. We would get $g^{\prime} x_{0}^{k}=x_{0}^{k} g^{\prime}$, thus $\phi^{k}\left(g^{\prime}\right)=g^{\prime}$, but this would imply that $g^{\prime}=e$, hence that $g \in \Gamma_{0}$, but this is a contradiction.

Finally, set, for every integer $n>0: g_{n}=x_{1} \cdots x_{n}$. Then it is easy to see that the double classes $\Gamma_{0} g_{n} \Gamma_{0}$ are pairwise distinct.

\section{The natural action of $\boldsymbol{F}$ and the associated Krieger factor}

Let $\Gamma$ be a group acting (on the left) in a measure class preserving way on a standard probability space $(\Omega, \mu)$. Recall that the full group $[\Gamma]$ of the action of $\Gamma$ on $\Omega$ is the group of all automorphisms $T$ of $\Omega$ such that $T \omega \in \Gamma \omega$ for $\mu$-a.e. $\omega \in \Omega$. We also set

$$
[\Gamma]_{0}=\{T \in[\Gamma] ; \mu \circ T=\mu\},
$$

which is the subgroup of elements of $[\Gamma]$ that preserve $\mu$. The ratio set of the action of $\Gamma$ on $\Omega$ is the set $r(\Gamma)$ of all numbers $\lambda \geq 0$ such that, for every $\varepsilon>0$ and for every Borel subset $A$ with positive measure, there exists a subset $B$ of $A$ with positive measure and $g \in \Gamma$ such that $g B \subset A$ and

$$
\left|\frac{d \mu \circ g}{d \mu}(\omega)-\lambda\right|<\varepsilon \quad \forall \omega \in B
$$


Then $r(\Gamma)$ is a closed subset of $[0, \infty)$ and $r(\Gamma) \backslash\{0\}$ is a closed subgroup of the multiplicative group $\mathbb{R}_{+}^{*}$. Moreover, one has: $r(\Gamma)=r([\Gamma])$.

We consider here the natural action of $F$ on the interval $[0,1]$ gifted with Lebesgue measure $\mu$. Our goal is to prove that the action of $F$ is ergodic and to compute its ratio set.

PROPOSITION 4.1. The action of $F$ on $[0,1]$ is ergodic and its ratio set is

$$
r(F)=\{0\} \cup\left\{2^{k} ; k \in \mathbb{Z}\right\} .
$$

Let $R_{F}$ be the equivalence relation on $[0,1]$ defined by the action of $F$ : if $s, t \in$ $[0,1]$, then the pair $(s, t)$ belongs to $R_{F}$ if and only if $s$ and $t$ are in the same $F$ orbit. Let $M\left(R_{F}\right)$ be the associated Feldman-Moore factor [5]; it generalizes the group measure space construction of Murray and von Neumann for not necessarily free actions. Then the next result follows immediately from Proposition 4.1 and from [5, Proposition 2.11].

COROLLARY 4.2. $M\left(R_{F}\right)$ is a factor of type $\mathrm{III}_{1 / 2}$.

The proof of Proposition 4.1 is inspired by [4, Section 2].

Let $K$ be the following group of bijections of $[0,1]:$ a bijection $\varphi$ from $[0,1]$ to itself belongs to $K$ if and only if there exists a partition $0<a_{1}<\cdots<a_{n}<1$ of $[0,1]$ into dyadic rational numbers such that

(K1) $\varphi(t)=t$ for every $t \in\left[0, a_{1}\right) \cup\left[a_{n}, 1\right]$;

(K2) for every $1 \leq j \leq n-1$, there exists a dyadic rational number $\alpha_{j}$ such that $\varphi(t)=t+\alpha_{j}$ for every $t \in\left[a_{j}, a_{j+1}\right)$.

LEMMA 4.3. $K$ is a subgroup of $[F]_{0}$. In particular, $\mu \circ \varphi=\mu$ for every $\varphi \in K$.

PROOF. It suffices to prove that, if $a, b$ and $\alpha$ are rational dyadic numbers such that $0<a<b<1$ and $0<a+\alpha<b+\alpha<1$, then there exists $f \in F$ such that $f(t)=t+\alpha$ for every $a \leq t<b$. We apply Lemma 1.2 with $a_{1}=a, a_{2}=b$ and $b_{1}=a+\alpha, b_{2}=b+\alpha$ (and $\left.a_{0}=b_{0}=0, a_{3}=b_{3}=1\right)$ : there exists $g \in F$ such that $g\left(a_{j}\right)=b_{j}$ for $j=0, \ldots, 3$. If $g(t)=t+\alpha$ for $t \in[a, b)$, then set $f=g$. If not, set $f(t)=g(t)$ for $t \in[0, a) \cup[b, 1]$ and $f(t)=t+\alpha$ for $a \leq t<b$. Then $f \in F$.

PROOF OF PROPOSITION 4.1. In order to prove that the action of $F$ is ergodic, it suffices to show that the action of $K$ is. Indeed, Lemma 4.3 implies thus that the action of the full group $[F]$ is ergodic, and [4, Lemma 2.8] applies to show that the action of $F$ is, too. We argue as in the proof of [4, Lemma 2.1]. Thus, let $X_{0} \subset[0,1]$ 
be a Borel set such that $\varphi X_{0}=X_{0}$ for every $\varphi \in K$ and that $\mu\left(X_{0}\right)>0$. Define a measure $v$ on $[0,1]$ by

$$
\nu(B)=\frac{\mu\left(B \cap X_{0}\right)}{\mu\left(X_{0}\right)}
$$

for every Borel set $B$. We have for every $\varphi \in K$ and every Borel set $B$

$$
\begin{aligned}
\nu(\varphi B) & =\frac{\mu\left((\varphi B) \cap X_{0}\right)}{\mu\left(X_{0}\right)}=\frac{\mu\left(B \cap \varphi^{-1} X_{0}\right)}{\mu\left(X_{0}\right)} \\
& \leq \frac{\mu\left(B \cap X_{0}\right)}{\mu\left(X_{0}\right)}+\frac{\mu\left(B \cap\left(\varphi^{-1} X_{0} \backslash X_{0}\right)\right)}{\mu\left(X_{0}\right)} \\
& =\frac{\mu\left(B \cap X_{0}\right)}{\mu\left(X_{0}\right)}=v(B) .
\end{aligned}
$$

Hence $v(\varphi B)=v(B)$ for every $\varphi$. In particular, one has $v([a+\alpha, b+\alpha))=v([a, b))$ for all dyadic rational numbers $a, b$ and $\alpha$ such that $[a, b] \cup[a+\alpha, b+\alpha] \subset[0,1]$, and this gives

$$
v\left(\left[\frac{l}{2^{n}}, \frac{l+1}{2^{n}}\right)\right)=\frac{1}{2^{n}}
$$

for every positive integer $n$ and every integer $0 \leq l \leq n$. Uniqueness of $\mu$ implies that $\nu=\mu$, and that $\mu\left(X_{0}\right)=1$. This proves ergodicity of the action of $K$.

It remains to compute $r(F)$. Denote by $\Gamma$ the group generated by $F$ and $K$. One has $[\Gamma]=[F]$, since $K \subset[F]$, and $r(\Gamma)=r(F)$.

As $(d \mu \circ g)(t) / d \mu \in\left\{2^{k} ; k \in \mathbb{Z}\right\}$ for $\mu$-a.e. $t \in[0,1]$ and for every $g \in F$, one has $r(F) \subset\{0\} \cup\left\{2^{k} ; k \in \mathbb{Z}\right\}$. As $r(F)$ is closed, the proof will be complete if we show that $2^{k} \in r(F)$ for every integer $k$. Then fix such a $k$ and choose $g \in F$ and dyadic rational numbers $0<a<b<1$ such that

$$
\frac{d \mu \circ g}{d \mu}(t)=\frac{d g}{d t}(t)=2^{k}
$$

for every $t \in(a, b)$. Let $A \subset[0,1]$ be a Borel set with positive measure. As the action of $K$ is ergodic, there exist $\varphi$ and $\psi \in K$ such that the Borel set

$$
B=\{t \in A ; \psi(t) \in(a, b) \text { and } \varphi g \psi(t) \in A\}
$$

has positive measure (see the proof of [4, Proposition 3.3]). Set $h=\varphi g \psi \in \Gamma$. One has $B \cup h B \subset A$ and

$$
\frac{d \mu \circ h}{d \mu}(t)=\frac{d \mu \circ g}{d \mu}(\psi(t))=2^{k}
$$

for every $t \in B$ since $\psi(t) \in(a, b)$. Hence $2^{k} \in r(F)$. 
REMARK. In fact, condition (K1) shows that $K$ is a subgroup of the full group of the commutator subgroup $F^{\prime}$. Hence the latter acts ergodically on $[0,1]$ as well, and it has the same ratio set as $F$. However, it gives the same equivalence relation on $[0,1]$, and thus the same factor $M\left(R_{F}\right)$.

\section{References}

[1] K. S. Brown and R. R. Geoghegan, 'An infinite-dimensional torsion-free $F P_{\infty}$ group', Invent. Math. 77 (1984), 367-381.

[2] J. W. Cannon, W. J. Floyd and W. R. Parry, 'Introductory notes on Richard Thompson's groups', Enseign. Math. 42 (1996), 215-256.

[3] M. Choda, 'A condition to construct a full $\mathrm{II}_{1}$-factor with an application to approximate normalcy', Math. Japon. 28 (1983), 383-398.

[4] P. Cutting and G. Robertson, 'Type III actions on boundaries of $\tilde{A}_{n}$ buildings', J. Operator Theory 49 (2003), 25-44.

[5] J. Feldman and C. C. Moore, 'Ergodic equivalence relations, cohomology and von Neumann algebras II', Trans. Amer. Math. Soc. 234 (1977), 325-359.

[6] P. Jolissaint, 'Moyennabilité intérieure du groupe F de Thompson', C. R. Math. Acad. Sci. Paris 325 (1997), 61-64.

[7] —- 'Central sequences in the factor associated with Thompson's group $F$ ', Ann. Inst. Fourier (Grenoble) 48 (1998), 1093-1106.

[8] G. Robertson, A. M. Sinclair and R. R. Smith, 'Strong singularity for subalgebras of finite factors', Internat. J. Math. 14 (2003), 235-258.

[9] G. Robertson and T. Steger, 'Maximal subalgebras of the group factor of an $\tilde{A}_{2}$ group', J. Operator Theory 36 (1996), 317-334.

[10] G. Zeller-Meier, 'Deux autres facteurs de type $\mathrm{II}_{1}$ ', Invent. Math. 7 (1969), 235-242.

Institut de Mathémathiques

Université de Neuchâtel

Emile-Argand 11

CH-2000 Neuchâtel

Switzerland

e-mail: paul.jolissaint@unine.ch 